\title{
A Comparison of Grammatical Bee Colony and Neural Networks in Medical Data Mining
}

\author{
Tapas Si \\ Assistant Professor \\ Department of Computer Science \& Engineering \\ Bankura Unnayani Institute of Engineering \\ Bankura, West Bengal, India
}

\author{
Sk. Sujauddin \\ Lead - Quality Assurance \\ Mindteck India Ltd. \\ Millennium City Information Technology Park, Tower II \\ Sector V, Salt Lake, Kolkata 700091, India
}

\begin{abstract}
This paper proposes a novel application of Grammatical Bee Colony for classification of medical data. Grammatical Bee Colony is a Swarm Programming algorithm generally used for automatic computer program generation in any arbitrary language. In this paper, Grammatical Bee Colony based classifier is designed and applied in medical data mining. The proposed method is applied on ten medical data sets and obtained results are compared with Multi-Layer Perceptron classifier trained with Levenberg-Marquardt algorithm. The proposed method statistically outperforms other method.
\end{abstract}

\section{General Terms}

Medical Data Mining, Machine Learning

\section{Keywords}

Medical data mining, Machine learning, Classification, Swarm programming, Grammatical bee colony, Artificial neural network

\section{INTRODUCTION}

Medical data mining is an important research area in medicine during past several years. Classification is one of the major clinical tasks in diagnosis of new disease. And classification tasks are also useful in the study of the different patterns in the medical data. The objective of classification of medical data is to predict the diagnostic decision (positive or negative). Artificial Neural Network (ANN) is a much popular machine learning tool and it is widely used in diagnostic classification of patients [1, 2]. ANN can handle diverse types of medical data and integrate them into categorized outputs [2]. In this paper, Grammatical Bee Colony (GBC) algorithm is used in classification of well-known medical data. T. Si et al. [3] proposed GBC algorithm and it is a Swarm Programming(SP) algorithm, generally used to generate computer programs in any arbitrary language. In GBC algorithm, Artificial Bee Colony $(\mathrm{ABC})$ is used as a learning algorithm in mapping from genotype (integer codons) to phenotype (computer program) while evolving the computer programs. In this paper, a novel application of GBC is presented as a binary classifier for medical data. The proposed GBC classifier is applied on ten medical data sets and a comparative study has been made with Multi-Layer Perceptron (MLP) trained using Levenberg-Marquardt (LM) method [2]. The GBC classifier statistically outperforms MLP classifier.

\section{PROPOSED GBC CLASSIFIER}

GBC is a Swarm Programming algorithm, and it is used in automatic program generation in any arbitrary language. $\mathrm{ABC}$ algorithm is used as a search engine in GBC. The computer programs are evolved using Backus-Naur Form (BNF) of Context-Free Grammar (CFG) through genotype-to-phenotype mapping. Genotype is the set of integer codons i.e food source's position and phenotype is the evolved computer program. The readers are encouraged to go through the paper [3] for details of GBC algorithm. The preliminary steps of the GBC classifier is the defining appropriate Context-Free Grammar in BNF for the data set to be classified. The role of GBC is to evolve computer program that computes a function $Y=f(X)$ where $X$ is the set of input attributes in the data set. The class label is determined using a logistic sigmoid function followed by a threshold function. The GBC classifier is depicted in Fig. 11 In next, Context-Free Grammar in BNF is described.

\subsection{Context-Free Grammar}

Context-Free Grammar in BNF is used in genotype-to-phenotype mapping. BNF of CFG for generating computer program in MAT$\mathrm{LAB}$ language is described below:

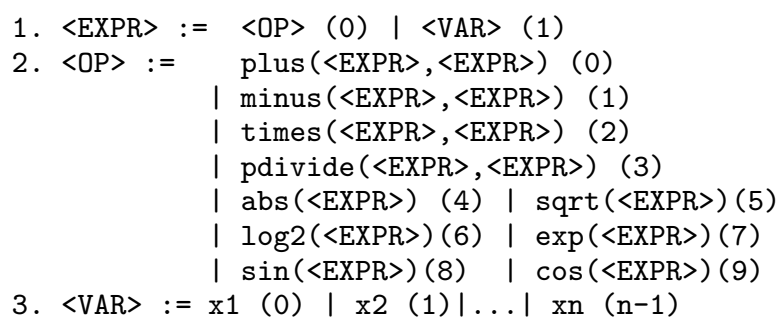

pdivide() is the protected division to avoid 'division-by-zero' error and $x 1, x 2, \ldots, x n$ are the input attributes in the data sets. 


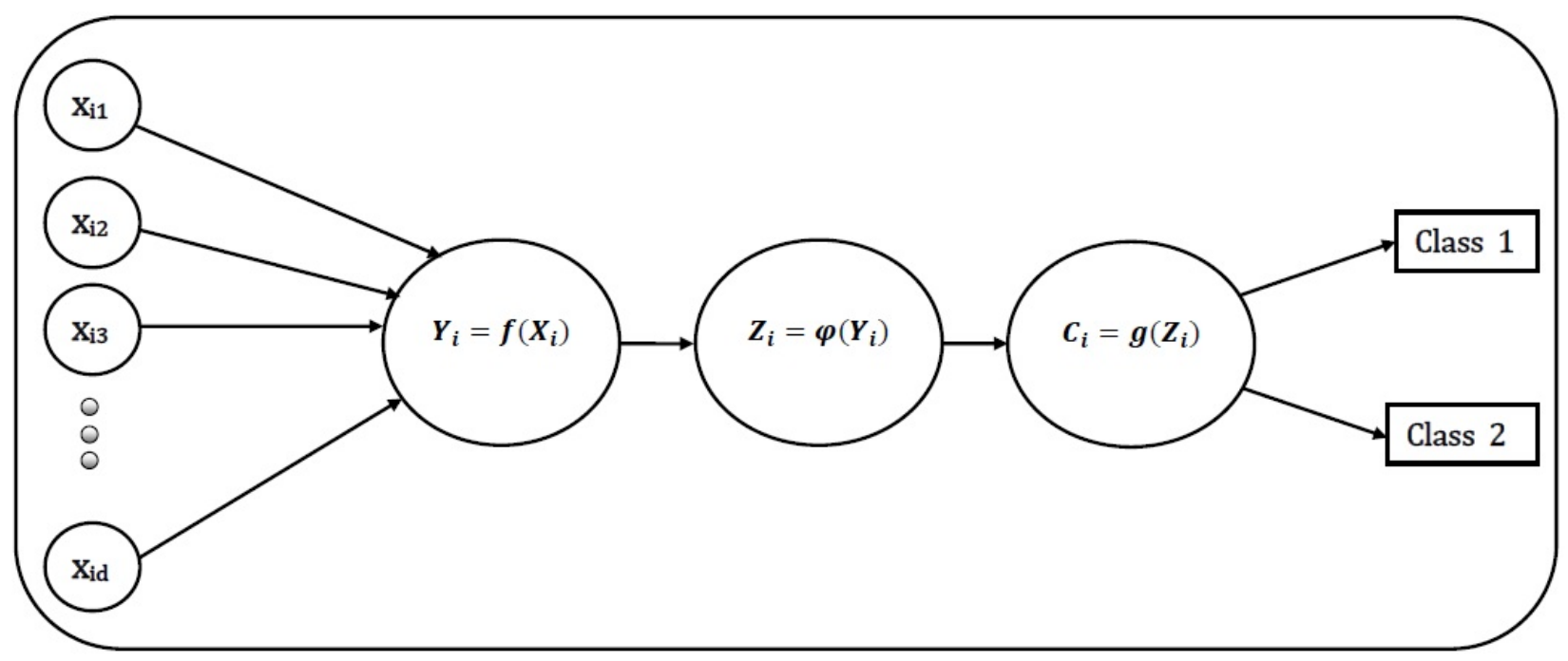

Fig. 1. GBC classifier.

\subsection{Genotype-to-Phenotype Mapping}

The food source position are generated in the range $[0,255]$ and rounded-off to its nearest integer values to form the codons. For an example, a part of genotype is given in Fig. 2

\begin{tabular}{|l|l|l|l|l|l|l|l|l|l|}
\hline 160 & 140 & 172 & 192 & 55 & 50 & 205 & 62 & 11 & 75 \\
\hline
\end{tabular}

Fig. 2. An example of a part of genotype

A mapping process is used in mapping from integer-valued food source position to the rule number in the derivation using CFG by the following ways:

Rule $=($ codon) MOD (number of choices for the current nonterminal)

If the current non-terminal $<$ EXPR $>$ is in derived string, then the rule number is generated as follows:

Rule $=(160 \bmod 2)=0$

$<$ EXPR $>$ will be replaced by $<\mathrm{OP}>$.

A complete derivation of phenotype from genotype in Fig. 2 is given below by assuming that the data set has $n=10$ attributes:

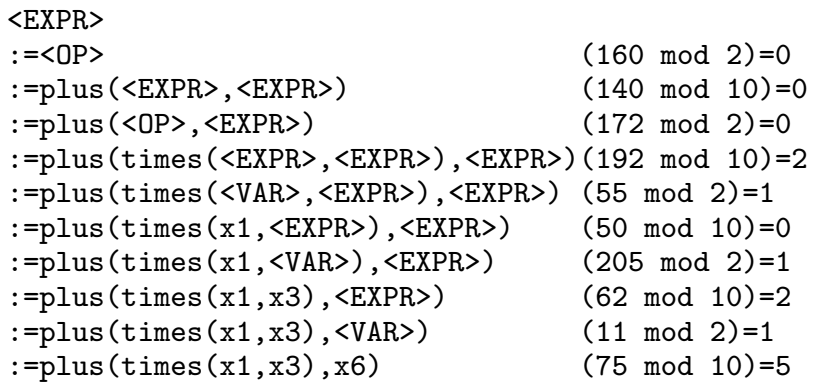

When derivation process runs out of codons, wrapping is carried out once.

\subsection{Classification}

The evolved computer programs are the functions $Y_{i}=f\left(X_{i}\right)$ discovered from the data set $X$ where $i=1,2,3, \ldots, M$. After evaluating the function $f\left(X_{i}\right)$, the output $Y_{i}$ for $i^{\text {th }}$ input $X_{i}$ is used to predict the class label $C_{i}, C_{i}=\{1,2\}$. A sigmoid function $\varphi\left(Y_{i}\right)$ is used in mapping from $Y_{i}$ to $Z_{i} \in(0,1)$ as follows:

$$
Z_{i}=\varphi\left(Y_{i}\right)=\frac{1}{1+e^{-Y_{i}}}
$$

Finally, class label $C_{i}$ is assigned to the input $X_{i}$ using a threshold function $g(\cdot)$ as following:

$$
C_{i}=g\left(Z_{i}\right)= \begin{cases}1 & Z_{i}<0.5 \\ 2 & Z_{i} \geq 0.5\end{cases}
$$

$C_{i}=1$ is positive class and $C_{i}=2$ is negative class in medical data set.

\subsection{Fitness Function}

In GBC algorithm, two objective functions are used such as misclassification rate (MR) and geometric mean $(\mathrm{GM})$ [6] over training data. GM measures the trade-off between the sensitivity and specificity by the following:

$$
G M=\sqrt{\text { sensitivity } \times \text { specificity }}
$$

A classifier is said to be good classifier if it provides higher classification accuracy and a good trade-off between sensitivity and specificity. The first objective function misclassification rate is to be minimized whereas second objective function geometric mean is to be maximized. The misclassification rate, sensitivity, specificity and GM values are measured using confusion matrix [5]. The two conflicting objective functions are transformed into a single objective function to be minimized as following:

$$
F=w_{1} \times M R+w_{2} \times(1-G M)
$$

Where $w_{1}, w_{2}$ are the weighting factor and $w_{1}=w_{2}=0.5$. The objective function $F$ is used as fitness function in GBC algorithm. 


\section{EXPERIMENTAL SETUP}

\subsection{Parameter Settings}

In $\mathrm{GBC}$, the parameters are set as following: population size $=100$, dimension $=100$, limit $=10$, maximum number of cycles $=500$. In LM algorithm, the maximum number of epochs is 2000 and threshold error is set to 0.001 , initial $\mu=0.001, \mu$ decrease and increase factors are 0.1 and 10 respectively, maximum $\mu=1 e 10$.

\subsection{PC Configuration}

(1) Operating System: Windows 7

(2) CPU: AMD FX -8150 Eight-Core $3.6 \mathrm{GHz}$

(3) RAM: 16 GB

(4) Software: Matlab 2010b

\section{RESULTS \& DISCUSSION}

The proposed Grammatical Bee Colony based classifier is applied on ten medical data sets such as Cleveland heart, liver disorders (BUPA), PIMA Indians Diabetes, Wisconsin diagnostic breast cancer (WDBC), Wisconsin prognostic breast cancer (WPBC), Lung Cancer, Vertebral column, Echocardiogram (ECG), SPECT heart, SPECTF heart. These data sets are collected from UCI Machine Learning Repository [4]. A short description of the data sets is given in Table 1 and the detail description is available from the Ref. [4]. The missing values in the data are replaced by attribute mean value [5] and the data are normalized in the range [0,1]. $\mathrm{K}-$ fold Cross-validation [5] is used for estimating generalization error of the proposed classifier and the value of $K$ is 10 in this work. The performance of GBC classifier is compared with MLP trained with LM algorithm (MLP-LM).

Table 1. Characteristic of Data sets

\begin{tabular}{|l|l|l|l|}
\hline Data Set & $\begin{array}{l}\text { Number of } \\
\text { patterns }\end{array}$ & $\begin{array}{l}\text { Number of } \\
\text { features }\end{array}$ & $\begin{array}{l}\text { Number of } \\
\text { classes }\end{array}$ \\
\hline Cleveland Heart & 303 & 14 & 2 \\
\hline Liver Disorder & 345 & 7 & 2 \\
\hline PIMA Diabetes & 768 & 8 & 2 \\
\hline WPBC & 198 & 34 & 2 \\
\hline WDBC & 569 & 32 & 2 \\
\hline Vertebral & 310 & 6 & 2 \\
\hline ECG & 131 & 13 & 2 \\
\hline SPECT & 267 & 22 & 2 \\
\hline SPECTF & 267 & 44 & 2 \\
\hline Lung Cancer & 32 & 57 & 3 \\
\hline
\end{tabular}

The mean and standard deviation of training accuracies and mean of CPU time for training are given in Table 2 The mean and standard deviation of testing accuracies are given in Table 3 Wilcoxon Signed Ranks Test [7] is carried out using mean testing accuracy values to compare the performance of the GBC classifier with MLP-LM classifier. The sum of positive ranks $\sum R^{+}$is 53.00 and sum of negative ranks $\sum R^{-}$is 2.00 . The obtain $p-$ value is 0.009344 which is lower than the significance level 0.01. This $p$-value is also much lower than the significance level 0.05. From this analysis, it has been seen that the proposed classifier statistically outperforms MLP-LM classifier. Though it is observed from Table 1 that training performance of MLP-LM classifier is higher than that of GBC classifier. To compare the class wise classification accuracy of data, sensitivity, specificity and GM measure are
Table 2. Mean and standard deviation of training accuracy and mean CPU time for training.

\begin{tabular}{|l|l|l|l|l|}
\hline \multirow{2}{*}{ Data Set } & \multicolumn{2}{|c|}{ CGBC } & \multicolumn{2}{c|}{ MLP-LM } \\
\cline { 2 - 5 } & Mean \pm std. & Time & Mean \pm std. & Time \\
\hline Cleveland Heart & $85.00 \pm 0.70$ & 13.33 & $99.60 \pm 0.32$ & 0.30 \\
\hline Liver Disorder & $68.71 \pm 1.08$ & 13.03 & $92.09 \pm 0.43$ & 0.93 \\
\hline PIMA Diabetes & $75.24 \pm 0.50$ & 12.48 & $92.74 \pm 0.38$ & 1.86 \\
\hline WPBC & $75.78 \pm 0.52$ & 12.11 & $100.00 \pm 0.00$ & 0.14 \\
\hline WDBC & $93.84 \pm 0.37$ & 13.89 & $100.00 \pm 0.00$ & 0.32 \\
\hline Vertebral & $80.47 \pm 1.69$ & 10.48 & $42.64 \pm 0.64$ & 0.96 \\
\hline ECG & $87.08 \pm 0.42$ & 11.69 & $92.42 \pm 0.83$ & 2.50 \\
\hline SPECT & $82.67 \pm 0.69$ & 13.16 & $94.16 \pm 0.17$ & 0.06 \\
\hline SPECTF & $79.05 \pm 1.02$ & 14.29 & $100.00 \pm 0.00$ & 0.57 \\
\hline Lung Cancer & $97.88 \pm 0.79$ & 12.55 & $100.00 \pm 0.00$ & 0.43 \\
\hline
\end{tabular}

Table 3. Mean and standard deviation of testing accuracy.

\begin{tabular}{|l|l|l|}
\hline Data Set & CGBC & MLP-LM \\
\hline Cleveland Heart & $79.81 \pm 0.63$ & $73.63 \pm 4.26$ \\
\hline Liver Disorder & $68.25 \pm 2.94$ & $67.17 \pm 4.97$ \\
\hline PIMA Diabetes & $77.47 \pm 4.05$ & $69.11 \pm 1.80$ \\
\hline WPBC & $70.91 \pm 1.10$ & $65.95 \pm 6.29$ \\
\hline WDBC & $92.06 \pm 1.38$ & $94.48 \pm 1.53$ \\
\hline Vertebral & $76.97 \pm 3.38$ & $39.81 \pm 1.96$ \\
\hline ECG & $84.84 \pm 4.19$ & $77.81 \pm 4.55$ \\
\hline SPECT & $79.05 \pm 2.26$ & $71.90 \pm 2.49$ \\
\hline SPECTF & $67.68 \pm 2.24$ & $63.01 \pm 3.31$ \\
\hline Lung Cancer & $90.37 \pm 5.81$ & $55.79 \pm 12.59$ \\
\hline
\end{tabular}

used and tabulated in Table 4 GM measures the trade-off between the sensitivity and specificity. The GBC classifier performs better than MLP-LM classifier in class wise classification for all except WDBC data set. The computation time of GBC classifier is higher in training than that of MLP-LM classifier. The derivation of phenotype from the genotype of each individual in GBC with one time wrapping takes higher computational time. It is also observed that all the codons in genotype are not used during the derivation process. Derivation stops when all the symbols in the derived string are terminals. Some codons are left as unused. Optimal use of genotype's length can reduce the computational time of GBC for training.

The GBC evolved programs for each fold with training and testing accuracy in classification of Lung cancer data are given in Table 5 From this table, it is observed that GBC evolves different programs for different folds. It is also observed that all the 56 attributes in Lung cancer data set are not used in the evolved program for the same fold. These different programs represent different functional relationships of output class with the input attributes in data set. From this discussion, it may be concluded that GBC is able to discover the knowledge about the relationship of patterns with its associated classes in the data set.

The above analysis of results demonstrates that the proposed GBC classifier is effective in classification of medical data. It also efficient in knowledge discovery from the medical data.

\section{CONCLUSIONS}

A novel application of Grammatical Bee Colony in medical data mining is proposed in this paper. The Grammatical Bee Colony classifier applied for classification of medical data sets and obtained results are compared with MLP trained using LM method. The pro- 
Table 4. Mean sensitivity, specificity and GM.

\begin{tabular}{|c|c|c|c|c|c|c|}
\hline \multirow[t]{2}{*}{ Data Set } & \multicolumn{3}{|c|}{ CGBC } & \multicolumn{3}{|c|}{ MLP-LM } \\
\hline & Sensitivity & Specificity & GM & Sensitivity & Specificity & GM \\
\hline $\begin{array}{l}\text { Cleveland } \\
\text { Heart }\end{array}$ & 74.43 & 84.39 & 79.17 & 67.65 & 78.73 & 72.95 \\
\hline $\begin{array}{l}\text { Liver } \\
\text { Disorder }\end{array}$ & 73.50 & 61.03 & 66.94 & 72.46 & 59.82 & 65.79 \\
\hline Diabetes & 71.46 & 80.69 & 75.91 & 55.46 & 76.47 & 65.10 \\
\hline WBCP & 77.58 & 49.53 & 61.87 & 72.83 & 44.16 & 56.53 \\
\hline WBCD & 91.56 & 92.89 & 92.21 & 97.66 & 89.14 & 93.28 \\
\hline Vertebral & 82.33 & 74.42 & 78.23 & 95.71 & 0.00 & 0.00 \\
\hline ECG & 71.02 & 91.50 & 80.49 & 68.52 & 82.52 & 75.16 \\
\hline SPECT & 83.57 & 61.85 & 71.24 & 77.52 & 50.10 & 62.27 \\
\hline SPECTF & 71.11 & 54.65 & 62.05 & 68.07 & 43.29 & 54.25 \\
\hline $\begin{array}{l}\text { Lung } \\
\text { Cancer }\end{array}$ & 88.92 & 98.00 & 93.24 & 62.91 & 20.33 & 31.03 \\
\hline
\end{tabular}

Table 5. GBC evolved programs of each fold with training and testing accuracy for Lung cancer.

\begin{tabular}{|c|c|c|c|}
\hline Fold\# & Program & Training accuracy & Testing Accuracy \\
\hline 1 & $\begin{array}{l}\text { times }(x 48, \operatorname{pdivide}(\operatorname{minus}(x 24, \operatorname{pdivide}(\cos (\exp (\sin (\operatorname{abs}(\operatorname{sqrt}(x 27))))) \\
\text {,plus }(\operatorname{pdivide}(\exp (x 4), x 14), \operatorname{minus}(x 26, x 4)))), \operatorname{plus}(\operatorname{sqrt}(x 13), x 27)))\end{array}$ & 96.43 & 100.00 \\
\hline 2 & $\begin{array}{l}\text { plus }(\exp (\cos (\operatorname{pdivide}(\exp (\sin (x 33)), \log 2(x 20)))) \\
\text { pdivide }(\operatorname{minus}(x 23, x 49), x 21))\end{array}$ & 96.49 & 100.00 \\
\hline 3 & times $(x 32$, minus $(x 26$, times $(x 54, x 1)))$ & 97.65 & 90.91 \\
\hline 4 & log2(plus (plus $(x 56$, abs (minus $(x 21, x 10)))$, times $(x 8, x 45)))$ & 98.23 & 93.33 \\
\hline 5 & $\begin{array}{l}\sin (\cos (\log 2(\text { times }(\operatorname{plus}(\operatorname{abs}(\operatorname{minus}(x 34, x 23)), x 1), \text { pdivide } \\
(\text { times }(x 40, \text { times }(\operatorname{plus}(x 20, \exp (\operatorname{abs}(x 43))), x 31)), x 39)))))\end{array}$ & 98.59 & 83.33 \\
\hline 6 & abs (pdivide $(x 56, x 13))$ & 98.26 & 85.00 \\
\hline 7 & $\begin{array}{l}\text { plus }(\sin (\operatorname{abs}(\exp (\log 2(\operatorname{minus}(\cos (x 38), x 3))))), \cos (\operatorname{minus}(x 31, \text { minus } \\
(\operatorname{pdivide}(x 56, x 23), \sin (\operatorname{csqrt}(x 31))))))\end{array}$ & 98.52 & 86.36 \\
\hline 8 & $\begin{array}{l}\text { minus (plus }(x 51, \exp (\cos (x 44))), \operatorname{plus}(\operatorname{abs}(\cos (\operatorname{plus}(x 22, \text { pdivide } \\
(\operatorname{plus}(\operatorname{abs}(x 50), x 51), \sin (\operatorname{pdivide}(\operatorname{abs}(x 13), \sin (x 56))))))), x 3))\end{array}$ & 98.27 & 88.00 \\
\hline 9 & $\begin{array}{l}\text { minus }(x 32, \operatorname{minus}(\sin (\mathrm{x} 31), \operatorname{minus}(\mathrm{x} 13, \operatorname{minus}(\operatorname{abs}(\operatorname{minus}(\mathrm{x} 30, \\
\exp (\operatorname{plus}(\operatorname{minus}(\mathrm{x} 14, \operatorname{pdivide}(\mathrm{x} 17, \mathrm{x} 18))), \sin (\log 2(\operatorname{plus} \\
(\mathrm{x} 2, \operatorname{pdivide}(\operatorname{minus}(\mathrm{x} 19, \mathrm{x} 49), \mathrm{x} 50))))))), \mathrm{x} 56))))\end{array}$ & 98.08 & 89.29 \\
\hline 10 & $\cos (\operatorname{sqrt}(\operatorname{minus}(\mathrm{x} 32, \operatorname{minus}(\operatorname{sqrt}(\operatorname{minus}(\mathrm{x} 13, \mathrm{x} 33)), \mathrm{x} 36))))$ & 98.26 & 87.50 \\
\hline
\end{tabular}

posed GBC classifier statistically outperforms MLP. The computational time of GBC classifier for training is higher than that of MLP. The performance improvement of the proposed GBC classifier will be the future work of this paper. In this paper, GBC algorithm is used for binary classification of medical data. This work can also be extended by the development of multi-class classifier using GBC algorithm.

\section{REFERENCES}

[1] J. Iavindrasana, G. Cohen, A. Depeursinge, H. Muller, R. Meyer and A. Geissbuhler, Clinical Data Mining: A Review, IMIA Yearbook of Medical Informatics, 2009.

[2] F. Amato et al.: Artificial neural networks in medical diagnosis, J Appl Biomed., Vol. 11, pp. 47-58, 2013. DOI:10.2478/v10136-012-0031-x

[3] T. Si, A. De, A.K. Bhattacharjee: Grammatical Bee Colony, In: B.K. Panigrahi et al. (Eds.): SEMCCO 2013, Part I, LNCS 8297, 2013, pp. 436-445

[4] http://cml.ics.uci.edu.

[5] L. Han, M. Kamber: Data Mining: Concepts and Techniques, Second Edition, Morgan Kaufmann Publishers, 2006.
[6] R. Barandela, J.S. Sanchez, V. Garc' a and E. Rangel, Strategies for learning in class imbalance problems, Pattern Recognition, pp. 849-851, 2003.

[7] J. Derrac, S. Garcia, D. Molina, F. Herrera, A practical tutorial on the use of nonparametric statistical tests as a methodology for comparing evolutionary and swarm intelligence algorithms, Swarm and Evolutionary Computation, Vol. 1, pp. 3$18,2011$. 